\title{
Comparative genetic characterization of Listeria monocytogenes isolates from human and animal listeriosis cases
}

\author{
Gregory T. Jeffers, ${ }^{1}$ James L. Bruce, ${ }^{2}$ Patrick L. McDonough, ${ }^{3}$ \\ Janet Scarlett, ${ }^{3}$ Kathryn J. Boor ${ }^{1}$ and Martin Wiedmann ${ }^{1}$
}

1 Department of Food Science, 412 Stocking Hall, Cornell University, Ithaca, NY 14853 , USA

2 Qualicon Inc., Wilmington, DE 19880, USA

3 Department of Population Medicine and Diagnostic Sciences, Cornell University, Ithaca, NY 14853, USA

Author for correspondence: Martin Wiedmann. Tel: +1 607254 2838. Fax: +1 6072544868. e-mail:mw16@cornell.edu

Listeria monocytogenes isolates from human sporadic and epidemic cases $(n=119)$ and from animal cases $(n=76)$ were characterized by automated ribotyping and PCR-restriction fragment length polymorphism (PCR-RFLP) typing of the virulence genes actA and $h / y$. This combination of typing methods differentiated 39 distinctive strains, each reflecting a unique combination of ribotypes, hly and actA alleles. Simpson's index of discrimination indicated a high discriminatory ability of ribotyping for both animal $(0.867)$ and human isolates $(0.857)$, which was further increased by the addition of $h / y$ and actA typing (0.916 and 0.904, respectively). Ribotype and $h / y$ allele data were further used to group isolates into three genetically distinct lineages. Each lineage is composed of several ribotype fragment subsets, each of which contains multiple ribotypes characterized by common ribotype fragments. To determine whether certain clones of $L$. monocytogenes show indications for unique pathogenic potential or host specificity, frequency distributions for five genetic characteristics (i.e. lineage, ribotype, ribotype fragment subset and $h / y$ and actA allele) were calculated for isolates from animal cases, human epidemic cases and human sporadic cases. Lineage III isolates were found less frequently in human cases (1 of 119 isolates) than in animal cases ( 8 of 76 isolates; $P=0.003$ ). These results suggest the possibility of host specificity for non-primate mammals among lineage III strains. In addition, lineage I strains were found more frequently among human cases than among animal cases $(P<0.001)$. Among the eight $h l y$ alleles observed, $h l y$ allele 1 was more common among human isolates as compared to animal isolates $(P=0.002)$. We also identified one ribotype (DUP-1030) which was significantly more common among animal isolates $(P=0.005)$ and one ribotype (DUP-1038; lineage I) which was significantly more common among human epidemic isolates as compared to human sporadic isolates $(P<0.001)$. These findings confirm the presence of clonal groups of $L$. monocytogenes, which appear to be characterized by unique virulence or host specificity patterns. This study also establishes baseline data describing the genetic diversity of human and animal $L$. monocytogenes isolates which can be utilized in future surveillance programmes to track the emergence of new strains.

Keywords: ribotyping, lineages, subtyping, pathogenic potential

\section{INTRODUCTION}

Listeria monocytogenes is a Gram-positive, facultative intracellular bacterium, which is considered a zoonotic pathogen. The organism is predominantly transmitted to humans by the food-borne route (Farber \& Peterkin, 1991). Recent estimates suggest that approximately 2500 human listeriosis cases occur annually in the US alone; these cases lead to about 500 deaths (Mead et al., 1999). 
Table 1. Human epidemic isolates

\begin{tabular}{|c|c|c|c|c|c|c|c|c|}
\hline \multirow[t]{2}{*}{ Outbreak (source) } & \multirow[t]{2}{*}{ Year } & \multirow[t]{2}{*}{ Isolate designation } & \multirow[t]{2}{*}{ Ribotype } & \multirow[t]{2}{*}{ Serotype } & \multicolumn{2}{|c|}{ Allele for: } & \multirow[t]{2}{*}{ Lineage } & \multirow[t]{2}{*}{ Reference } \\
\hline & & & & & $h l y$ & act $A$ & & \\
\hline Anjou, France & 1976 & FSL-J1-112/TS31/L4491a & 1038 & $4 \mathrm{~b}$ & 1 & 3 & I & Carbonelle et al. (1978) \\
\hline Boston, USA (raw vegetables) & 1979 & FSL-J1-220 & 1042 & $4 \mathrm{~b}$ & 1 & 4 & I & Ho et al. (1986) \\
\hline Carlisle, England & 1981 & FSL-J1-105/TS23/L745 & 1030 & $1 / 2 \mathrm{a}$ & 2 & 4 & II & McLauchlin et al. (1986) \\
\hline \multirow[t]{3}{*}{ Nova Scotia, Canada (coleslaw) } & 1981 & FSL-J1-003 & 1038 & $4 \mathrm{~b}$ & 1 & 3 & I & Schlech et al. (1983) \\
\hline & & FSL-J1-107/TS26/L4742 & 1038 & $4 \mathrm{~d}$ & 1 & 3 & I & \\
\hline & & FSL-J1-108/TS27/L4738 & 1038 & $4 \mathrm{~b}$ & $1 \mathrm{~b}$ & 3 & I & \\
\hline $\begin{array}{l}\text { Massachusetts, USA (pasteurized } \\
\text { milk) }\end{array}$ & 1983 & ScottA & 1042 & $4 \mathrm{~b}$ & 1 & 4 & I & Fleming et al. (1985) \\
\hline \multirow[t]{2}{*}{ United Kingdom (paté) } & $1988-1990$ & FSL-J1-116/TS38/L3306 & 1042 & $4 \mathrm{~b}$ & 1 & 4 & I & McLauchlin et al. (1991) \\
\hline & & FSL-J1-129/TS65/L3238 & 1042 & $4 \mathrm{bx}$ & 1 & 4 & I & \\
\hline \multirow[t]{2}{*}{ Los Angeles, USA (soft cheese) } & 1985 & FSL-J1-110/TS29/F2365 & 1038 & $4 \mathrm{~b}$ & 1 & 3 & I & Linnan et al. (1988) \\
\hline & & FSL-J1-002 & 1038 & $4 \mathrm{~b}$ & 1 & 4 & I & \\
\hline Switzerland (soft smear cheese) & $1983-1987$ & FSL-J1-123/TS55/L4486a & 1038 & $4 \mathrm{~b}$ & 1 & 3 & I & Bille (1990) \\
\hline \multirow{2}{*}{ Philadelphia, USA (ice cream, salami) } & 1987 & FSL-J1-012 & 1038 & & 1 & 3 & I & Schwartz et al. (1989) \\
\hline & & FSL-J1-020 & 1042 & & 1 & 4 & I & \\
\hline
\end{tabular}

Listeriosis symptoms in humans and animals include encephalitis, meningitis, septicaemia and abortion. Despite the fact that not all strains of L. monocytogenes are thought to be pathogenic to humans (Hof \& Rocourt, 1992), host specificity has yet to be demonstrated for different L. monocytogenes strains. Host specificities have been identified in other bacterial pathogens that affect both animals and humans. For example, host specificity has been suggested for either humans or cattle among different clones of Staphylococcus aureus (Kapur et al., 1995; Musser \& Selander, 1990). Different strains of Pasteurella haemolytica, the aetiological agent of pneumonic pasteurellosis, also appear to display host specificity for either cattle or sheep (Davies et al., 1997).

Several studies have suggested that L. monocytogenes may be characterized by a clonal structure and that pathogenic potentials may differ among clonal groups (Piffaretti et al., 1989; Wiedmann et al., 1997). Rasmussen et al. (1995) demonstrated that L. monocytogenes can be divided into three lineages based on sequence determination of the virulence genes bly, iap and the gene flaA encoding flagellin. Wiedmann et al. (1997) confirmed classification of L. monocytogenes into three genetically distinct lineages through ribotyping and PCR-RFLP of the virulence gene hly. Notably, all human epidemic isolates clustered into lineage I and none of the 21 human isolates examined fell into lineage III (Wiedmann et al., 1997). Isolates from human sporadic cases were distributed between lineage I and lineage II (65 and $35 \%$, respectively), while animal isolates appeared to encompass a more diverse population which spanned all three lineages (Wiedmann et al., 1997).

At the outset of this study we hypothesized (i) that the three genetic lineages or specific clonal groups of $L$. monocytogenes show unique pathogenic potentials for different hosts, namely humans and animals; (ii) that all lineage III strains, or a subset thereof, show attenuated virulence in humans; and (iii) that all lineage I strains, or a subset thereof, show unique pathogenic potential towards humans. This current study was designed to probe the clonal structure of L. monocytogenes and to evaluate the distribution of different clonal groups and virulence gene alleles among human and animal $L$. monocytogenes isolates to identify possible differences in pathogenic potentials and host specificities within this species. To that end, we have assembled a collection of 119 human and 76 animal $L$. monocytogenes isolates and have characterized these strains using automated ribotyping and PCR-RFLP typing of the virulence genes $a c t A$ and $h l y$. These data were analysed for the existence of distribution differences for specific genetic types and strains among human epidemic, human sporadic and animal listeriosis isolates. Specific clones or strains that differ in distribution to these groups are likely to differ from each other in their pathogenic potentials. Furthermore, our data were utilized to determine the discriminatory power of the typing methods used (i.e. automated ribotyping and PCR-RFLP of act $A$ and $h l y)$.

\section{METHODS}

Strains and isolates. A total of 119 human and 76 animal $L$. monocytogenes isolates from various sources were used in this study. Human isolates included those from sporadic cases as well as those from nine different epidemic outbreaks defined as such in the World Health Organization's (WHO) International Multicentre L. monocytogenes subtyping study (Table 1) (Bille \& Rocourt, 1996). Human isolates from sporadic cases were also obtained from the Sunnybrook Health Science Centre (University of Toronto, Ontario, Canada) as described by Louie et al. (1996), the Centers for Disease Control (Atlanta, Georgia), the Department of Health Services (Los Angeles, California) and Qualicon Inc. (Wilmington, Delaware). The majority of these human isolates were collected between 1982 and 1995. The animal isolate collection, previously described by Wiedmann et al. (1997), was obtained from the Diagnostic Laboratory, College of Veterinary Medicine, Cornell University. Sixty-four isolates were from ruminants (cattle, sheep and goats) and 10 were from non-ruminants (horses, primates and birds) (Wiedmann et al., 1997). 
All isolates were characterized by automated ribotyping and PCR-RFLP analysis of the virulence genes $a c t A$ and $b l y$. These data were used to classify isolates on the basis of the following five genetic characteristics: lineage, ribotype, ribotype fragment subset, hly allele and $a c t A$ allele. Isolate classification to lineage was designated based on ribotype and hly allele data as described by Wiedmann et al. (1997). Isolates were clustered into ribotype fragment subsets as described by Bruce et al. (1995).

Virulence gene allele characterization. Virulence gene alleles for $a c t A$ and $h l y$ were determined by PCR-RFLP as described previously (Wiedmann et al., 1997).

Ribotyping. A subset of isolates was manually ribotyped using the procedure described by Bruce et al. (1995) and reported by Wiedmann et al. (1997). The remaining samples and representatives from each of the reported ribotypes were then processed using the RiboPrinter Microbial Characterization System (Qualicon). This procedure was carried out to enable the use of standardized data for future reference. The RiboPrinter system process has been described by Bruce (1996). In brief, this automated process includes bacterial cell lysis and cleavage of the DNA using the restriction enzyme EcoRI. DNA fragments are separated by size using electrophoresis and analysed using a modified Southern hybridization blotting technique. The DNA is hybridized with a labelled rRNA operon probe derived from E. coli and the bands are detected using a chemiluminescent substrate. An image is captured using a customized charge-coupled device camera and is then electronically transferred to the system's computer. Each sample lane of data is normalized to a standard marker set, characterized and identified using similarity measurements to previously run isolates and reference patterns.

Simpson's index of discrimination (SID). The suitability of typing methods for differentiation of strains was determined using Simpson's numerical index as described by Hunter \& Gaston (1988).

Statistical methods. Isolates were separated into three categories (i.e. human epidemic, human sporadic and animal isolates), then frequency distributions were calculated separately for each genetic characteristic. Human epidemic and human sporadic isolates were also pooled to allow a comparison of human isolates versus animal isolates. The frequency distributions of each genetic characteristic between animal versus human and human epidemic versus human sporadic isolates were compared using a $\chi^{2}$ of independence test. For comparisons where one or more of the expected values were $<5$, Fisher's exact test was used. $P$ values $\leqslant 0.05$ were considered statistically significant. The significance level was left at $\leqslant 0.05$ and was not adjusted for the fact that multiple comparisons were made. We chose this approach to avoid missing possible associations of genetic characteristics (caused by a very conservative $P$ value) and provided observed $P$ values instead. With a Bonferroni correction for multiple comparisons $(n=83)$, a significant $P$ value would be approximately $P \leqslant 0 \cdot 0006$. Readers can evaluate the significance according to their preferred criteria.

\section{RESULTS}

\section{Genetic characterization of human and animal isolates}

Ribotypes and PCR-RFLP alleles for act $A$ and $b l y$ were determined for 119 human and 76 animal isolates (Table 2). These analyses designated 39 subtypes (A-AN),
Table 2. Human and animal isolates and their genetic characteristics

\begin{tabular}{|c|c|c|c|c|c|}
\hline \multirow{3}{*}{$\begin{array}{l}\text { Strain } \\
\text { type }\end{array}$} & \multirow[t]{3}{*}{ Ribotype } & \multicolumn{2}{|c|}{ Allele for: } & \multirow{2}{*}{\multicolumn{2}{|c|}{$\begin{array}{c}\text { No. of isolates for each } \\
\text { strain type obtained } \\
\text { from: }\end{array}$}} \\
\hline & & \multirow[t]{2}{*}{ act $A$} & \multirow[t]{2}{*}{$h l y$} & & \\
\hline & & & & $\begin{array}{l}\text { Humans } \\
(n=119)\end{array}$ & $\begin{array}{l}\text { Animals } \\
(n=76)\end{array}$ \\
\hline $\mathrm{A}$ & DUP-1024 & 3 & 1 & 4 & 0 \\
\hline $\mathrm{B}$ & DUP-1024 & 4 & 1 & 1 & 0 \\
\hline $\mathrm{C}$ & DUP-1026 & 3 & 1 & 1 & 0 \\
\hline $\mathrm{D}$ & DUP-1027 & 3 & 1 & 3 & 0 \\
\hline $\mathrm{E}$ & DUP-1027 & 3 & $1 \mathrm{~b}$ & 1 & 0 \\
\hline $\mathrm{F}$ & DUP-1029 & 4 & 2 & 2 & 0 \\
\hline G & DUP-1030 & 3 & 2 & 1 & 3 \\
\hline $\mathrm{H}$ & DUP-1030 & 4 & 2 & 12 & 17 \\
\hline I & DUP-1030 & 4 & $2 c$ & 0 & 1 \\
\hline $\mathrm{J}$ & DUP-1035 & 4 & 2 & 1 & 0 \\
\hline $\mathrm{K}$ & DUP-1038 & 3 & 1 & 17 & 9 \\
\hline $\mathrm{L}$ & DUP-1038 & 3 & $1 b$ & 1 & 0 \\
\hline M & DUP-1038 & 4 & 1 & 1 & 0 \\
\hline $\mathrm{N}$ & DUP-1039 & 3 & 2 & 1 & 0 \\
\hline $\mathrm{O}$ & DUP-1039 & 4 & 2 & 4 & 1 \\
\hline $\mathrm{P}$ & DUP-1039 & 4 & $2 b$ & 0 & 1 \\
\hline Q & DUP-1040 & 4 & 1 & 0 & 1 \\
\hline $\mathrm{R}$ & DUP-1042 & 3 & 1 & 9 & 9 \\
\hline $\mathrm{T}$ & DUP-1042 & 4 & 1 & 28 & 6 \\
\hline $\mathrm{U}$ & DUP-1043 & 3 & 1 & 1 & 0 \\
\hline $\mathrm{V}$ & DUP-1044 & 3 & 1 & 2 & 4 \\
\hline W & DUP-1044 & 4 & 1 & 5 & 1 \\
\hline $\mathrm{X}$ & DUP-1045 & 3 & 2 & 0 & 1 \\
\hline $\mathrm{Y}$ & DUP-1045 & 4 & 2 & 4 & 6 \\
\hline $\mathrm{Z}$ & DUP-1047 & 4 & 2 & 1 & 3 \\
\hline AA & DUP-1052 & 3 & 1 & 4 & 2 \\
\hline $\mathrm{AB}$ & DUP-1052 & 4 & 1 & 1 & 0 \\
\hline $\mathrm{AC}$ & DUP-1053 & 4 & 2 & 4 & 0 \\
\hline $\mathrm{AD}$ & DUP-1054 & 4 & 2 & 0 & 1 \\
\hline $\mathrm{AE}$ & DUP-1056 & 4 & 2 & 2 & 0 \\
\hline $\mathrm{AF}$ & DUP-1059 & 4 & $1 b$ & 0 & 3 \\
\hline AG & DUP-1059 & 4 & $4 b$ & 1 & 1 \\
\hline $\mathrm{AH}$ & DUP-1061 & 4 & $4 b$ & 0 & 1 \\
\hline $\mathrm{AI}$ & DUP-1062 & 4 & 2 & 6 & 1 \\
\hline $\mathrm{AJ}$ & DUP-1062 & 4 & $2 b$ & 1 & 0 \\
\hline AK & DUP-10146 & 4 & $1 \mathrm{c}$ & 0 & 1 \\
\hline $\mathrm{AL}$ & DUP-10147 & 4 & $4 a$ & 0 & 1 \\
\hline $\mathrm{AM}$ & DUP-10144 & 4 & 2 & 0 & 1 \\
\hline $\mathrm{AN}$ & DUP-10145 & 4 & $4 a$ & 0 & 1 \\
\hline
\end{tabular}

which are characterized by unique combinations of ribotypes, act $A$ and hly alleles. Human and animal isolates comprised 28 and 24 subtypes, respectively. Fifteen and 11 subtypes were unique to either humans or animals, respectively. Eighteen ribotypes were found among the 119 human isolates, while 17 ribotypes were found among the 76 animal isolates.

The genetic characteristics of 14 human epidemic 
Table 3. Frequency distribution of Listeria monocytogenes types among animal, human epidemic and human sporadic isolates

\begin{tabular}{|c|c|c|c|c|c|}
\hline \multirow[t]{2}{*}{ Genetic strain types } & \multicolumn{3}{|c|}{ Number of isolates $(\%)$ from: } & \multicolumn{2}{|c|}{$P$ value of $\chi^{2}$ test for $:^{*}$} \\
\hline & $\begin{array}{l}\text { Animal cases } \\
\quad(n=76)\end{array}$ & $\begin{array}{c}\text { Human epidemic } \\
\text { cases } \\
(n=14)\end{array}$ & $\begin{array}{c}\text { Human sporadic } \\
\text { cases } \\
(n=105)\end{array}$ & $\begin{array}{l}\text { Animal cases vs } \\
\text { human cases }\end{array}$ & $\begin{array}{c}\text { Human epidemic } \\
\text { cases vs human } \\
\text { sporadic cases }\end{array}$ \\
\hline \multicolumn{6}{|l|}{$h l y$ alleles } \\
\hline 1 & $32(42 \cdot 1)$ & $12(85 \cdot 7)$ & $65(61 \cdot 9)$ & $0 \cdot 002 \dagger$ & $0 \cdot 134$ \\
\hline $1 b$ & $3(3 \cdot 9)$ & $1(7 \cdot 1)$ & $1(1 \cdot 0)$ & $0 \cdot 380$ & $0 \cdot 222$ \\
\hline $1 \mathrm{c}$ & $1(1 \cdot 3)$ & 0 & 0 & $0 \cdot 390$ & NA \\
\hline 2 & $34(44 \cdot 7)$ & $1(7 \cdot 1)$ & $37(35 \cdot 2)$ & $0 \cdot 071$ & $0 \cdot 063$ \\
\hline $2 b$ & $1(1 \cdot 3)$ & 0 & $1(1 \cdot 0)$ & $1 \cdot 000$ & $1 \cdot 000$ \\
\hline $2 c$ & $1(1 \cdot 3)$ & 0 & 0 & $0 \cdot 390$ & NA \\
\hline $4 \mathrm{a}$ & $2(2 \cdot 6)$ & 0 & 0 & $0 \cdot 151$ & NA \\
\hline $4 b$ & $2(2 \cdot 6)$ & 0 & $1(1 \cdot 0)$ & $0 \cdot 561$ & $1 \cdot 000$ \\
\hline \multicolumn{6}{|l|}{ act $A$ alleles } \\
\hline 3 & $28(36 \cdot 8)$ & $7(50 \cdot 0)$ & $38(36 \cdot 2)$ & $0 \cdot 891$ & $0 \cdot 317$ \\
\hline 4 & $48(63 \cdot 2)$ & $7(50 \cdot 0)$ & $67(63 \cdot 8)$ & $0 \cdot 891$ & $0 \cdot 317$ \\
\hline \multicolumn{6}{|l|}{ Lineages } \\
\hline I & $32(42 \cdot 1)$ & $13(92 \cdot 9)$ & $66(62 \cdot 9)$ & $8 \times 10^{-4} \dagger$ & $0.033 \ddagger$ \\
\hline II & $36(47 \cdot 4)$ & $1(7 \cdot 1)$ & $38(36 \cdot 2)$ & $0 \cdot 041 \neq$ & $0 \cdot 034 \ddagger$ \\
\hline III & $8(10 \cdot 5)$ & 0 & $1(1 \cdot 0)$ & $0 \cdot 003+$ & $1 \cdot 000$ \\
\hline \multicolumn{6}{|l|}{ Ribotype fragment subsets } \\
\hline E 11.2 & $11(14 \cdot 5)$ & $8(57 \cdot 1)$ & $20(19 \cdot 0)$ & $0 \cdot 123$ & $0 \cdot 004+$ \\
\hline E 5.2 & $28(36 \cdot 8)$ & $1(7 \cdot 1)$ & $31(29 \cdot 5)$ & $0 \cdot 142$ & $0 \cdot 109$ \\
\hline E 5.3 & $2(2 \cdot 6)$ & 0 & $5(4 \cdot 8)$ & $0 \cdot 707$ & $0 \cdot 634$ \\
\hline E $5.3,-\mathrm{D}$ & $4(5 \cdot 3)$ & 0 & $8(7 \cdot 6)$ & $0 \cdot 768$ & $0 \cdot 594$ \\
\hline E 9.2 & $18(23 \cdot 7)$ & $5(35 \cdot 7)$ & $38(36 \cdot 2)$ & $0 \cdot 067$ & $0 \cdot 972$ \\
\hline G 6.2, H 9.0 & $1(1 \cdot 3)$ & 0 & $1(1 \cdot 0)$ & $1 \cdot 000$ & $1 \cdot 000$ \\
\hline G $8.1, H 7.1$ & $8(10 \cdot 5)$ & 0 & $1(1 \cdot 0)$ & $0.003+$ & $1 \cdot 000$ \\
\hline Temperature-controlled & $4(5 \cdot 3)$ & 0 & $1(1 \cdot 0)$ & $0 \cdot 077$ & $1 \cdot 000$ \\
\hline \multicolumn{6}{|l|}{ Ribotype } \\
\hline 1024 & 0 & 0 & $5(4 \cdot 8)$ & $0 \cdot 159$ & $0 \cdot 634$ \\
\hline 1026 & 0 & 0 & $1(1 \cdot 0)$ & $1 \cdot 000$ & $1 \cdot 000$ \\
\hline 1027 & 0 & 0 & $4(3 \cdot 8)$ & $0 \cdot 158$ & $1 \cdot 000$ \\
\hline 1029 & 0 & 0 & $2(1.9)$ & 0.522 & $1 \cdot 000$ \\
\hline 1030 & $20(26 \cdot 3)$ & $1(7 \cdot 1)$ & $12(11 \cdot 4)$ & $0.005+$ & $0 \cdot 710$ \\
\hline 1035 & 0 & 0 & $1(1 \cdot 0)$ & $1 \cdot 000$ & $1 \cdot 000$ \\
\hline 1038 & $9(11 \cdot 8)$ & $8(57 \cdot 1)$ & $11(10 \cdot 5)$ & $0 \cdot 423$ & $2 \cdot 0 \times 10^{-6} \dagger$ \\
\hline 1039 & $2(2 \cdot 6)$ & 0 & $5(4 \cdot 8)$ & 0.707 & 0.634 \\
\hline 1040 & $1(1 \cdot 3)$ & 0 & 0 & $0 \cdot 390$ & NA \\
\hline 1042 & $15(19 \cdot 7)$ & $5(35 \cdot 7)$ & $32(30 \cdot 5)$ & $0 \cdot 080$ & $0 \cdot 761$ \\
\hline 1043 & 0 & 0 & $1(1 \cdot 0)$ & $1 \cdot 000$ & $1 \cdot 000$ \\
\hline 1044 & $6(7 \cdot 9)$ & 0 & $7(6 \cdot 7)$ & 0.583 & 0.598 \\
\hline 1045 & $7(9 \cdot 2)$ & 0 & $4(3 \cdot 8)$ & $0 \cdot 113$ & $1 \cdot 000$ \\
\hline 1047 & $3(3.9)$ & 0 & $1(1 \cdot 0)$ & $0 \cdot 301$ & $1 \cdot 000$ \\
\hline 1052 & $2(2 \cdot 6)$ & 0 & $5(4 \cdot 8)$ & $0 \cdot 707$ & 0.634 \\
\hline 1053 & 0 & 0 & $4(3 \cdot 8)$ & $0 \cdot 158$ & $1 \cdot 000$ \\
\hline 1054 & $1(1 \cdot 3)$ & 0 & 0 & $0 \cdot 390$ & NA \\
\hline 1056 & 0 & 0 & $2(1 \cdot 9)$ & 0.522 & $1 \cdot 000$ \\
\hline 1059 & $4(5 \cdot 3)$ & 0 & $1(1 \cdot 0)$ & 0.077 & $1 \cdot 000$ \\
\hline 1061 & $1(1 \cdot 3)$ & 0 & 0 & $0 \cdot 390$ & NA \\
\hline 1062 & $1(1 \cdot 3)$ & 0 & $7(6 \cdot 7)$ & $0 \cdot 153$ & 0.598 \\
\hline dd11696 & $1(1 \cdot 3)$ & 0 & 0 & $0 \cdot 390$ & NA \\
\hline dd11698 & $1(1 \cdot 3)$ & 0 & 0 & $0 \cdot 390$ & NA \\
\hline dd11900 & $1(1 \cdot 3)$ & 0 & 0 & $0 \cdot 390$ & NA \\
\hline dd11903 & $1(1 \cdot 3)$ & 0 & 0 & $0 \cdot 390$ & NA \\
\hline
\end{tabular}

$* \mathrm{NA}, \chi^{2}$ test not applicable.

$\dagger P$ values $\leqslant 0 \cdot 05$.

$\ddagger P$ values $\leqslant 0 \cdot 05$ which were $>0 \cdot 05$ when calculations were repeated counting only one single isolate of each genetic type for each of the outbreaks represented by multiple isolates (see Results). 
isolates from nine different outbreaks are presented separately in Table 1 . Multiple epidemic isolates were available for subtyping for four outbreaks (Nova Scotia, Canada, 1981; Los Angeles, USA, 1985; United Kingdom, 1988-1990; Philadelphia, USA, 1987). Multiple isolates from each of the four outbreaks differed by at least one genetic characteristic or by their serotype, suggesting that each represented a distinctive strain (Table 1). For example, one of the three isolates from the 1991 Nova Scotia outbreak was characterized by hly allele $1 \mathrm{~b}$ while the other two carried bly allele 1 . The two isolates from the 1985 Los Angeles outbreak in our collection carried different act $A$ alleles (Table 1). Since only two isolates from this outbreak were available for subtyping in our laboratory, the frequency of these two subtypes among all isolates from this outbreak is not known. For the outbreaks in the United Kingdom (1988-1990) and in Philadelphia (1987), different subtypes were shown to cause a significant number of cases (Schwartz et al., 1989; McLauchlin et al., 1991). The serotype $4 \mathrm{~d}$ strain isolated during the Nova Scotia outbreak (Table 1 ) appears to have caused only a few cases or a single case in this outbreak (Schlech et al., 1983). For the other outbreaks included in this study, a single L. monocytogenes subtype appears to have been responsible for all cases (see references in Table 1).

\section{Frequency distribution of $L$. monocytogenes genetic characteristics among human and animal isolates}

The information in Table 2 was used to determine the frequency distribution in each category (human epidemic, human sporadic and animal) of the five genetic characteristics ( $h$ ly alleles, act $A$ alleles, lineage, ribotype and ribotype fragment subset) determined for each isolate (Table 3). We chose to include each of the multiple isolates from a given outbreak in calculating the frequency distribution of the human epidemic isolates since each isolate appeared to represent a distinct strain (see above and Table 1). To account for this possible sampling bias, calculations were also repeated counting only one single isolate of each genetic type for each of the outbreaks represented by multiple isolates. For example, in these calculations, only one isolate from the Nova Scotia outbreak was included in the frequency distribution of lineages (as all three isolates belong to lineage I), while one bly allele 1 and one bly allele $1 \mathrm{~b}$ isolate were included in the frequency distribution of bly alleles. In the remainder of this paper, we will refer to these calculations as the "conservative calculation with epidemic duplicates removed'. Using these conservative calculations with epidemic duplicates removed, five $P$ values which previously indicated significant differences with $P$ values between 0.02 and 0.05 changed to nonsignificant levels $(P>0.05)$ (Table 3$)$. These $P$ values are indicated by $\neq$ in Table 3 , while $P$ values with significant differences in both calculations are indicated by $t$. Only frequency distributions that showed significant differences in both calculations were considered to reflect robust differences in frequency of the respective genetic types.

Lineage III strains were isolated less frequently from human cases $(0.8 \%)$ than from animal cases $(10.5 \%)$. Lineage I strains represented $92.9 \%$ of human epidemic and $62.9 \%$ of human sporadic isolates and were significantly more frequent among human cases as compared to animal cases $(42 \cdot 1 \%)$. Animal isolates were classified into lineages I and II with similar frequencies (42.1 and $47 \cdot 4 \%$, respectively).

Ribotype fragment subsets E 11.2, E 5.2 and E 9.2 represent the most frequently isolated ribotype fragment subsets from humans and animals $(82 \cdot 1 \%$ of all isolates). Ribotype fragment subset G 8.1, H 7.1, one of three subsets constituting lineage III (Wiedmann et al., 1997), was significantly less frequently isolated from humans $(0.8 \%)$ than from animals $(10.5 \%)$. Subset E 11.2 was significantly more common among the epidemic isolates $(57.1 \%)$ than among the human sporadic isolates $(19.0 \% ; P=0.004)$. The $P$ value for the comparison of human epidemic and sporadic isolates increased to $P=$ 0.038 in the conservative calculation with epidemic duplicates removed.

Ribotypes DUP-1030 and DUP-1038 are the only ribotypes that showed significant differences in their frequencies among human epidemic, human sporadic and animal cases. DUP-1030 was more frequently associated with animal cases $(26.3 \%)$ than with human cases $(11 \cdot 4 \%)$. DUP-1038 was more frequent among human epidemic isolates $(57 \cdot 1 \%)$ than among human sporadic isolates $\left(10.5 \% ; P=2.0 \times 10^{-6}\right)$. This $P$ value increased to $P=0.0046$ in the conservative calculation with epidemic duplicates removed. DUP-1042 is the most common ribotype accounting for $35.7 \%$ of the human epidemic isolates and for $30.5 \%$ of the sporadic isolates, but for only $19 \cdot 7 \%$ of all animal isolates.

PCR-RFLP analysis of the hly gene allowed us to differentiate eight hly alleles among the 195 isolates. hly alleles 1 and 2 were the two most common bly alleles. bly allele 1 represented $64.7 \%$ of all human isolates (epidemic and sporadic) and was significantly more common among human as compared to animal isolates $(42 \cdot 1 \%)$. hly allele $1 \mathrm{~b}$ was found in two lineage I human isolates as well as in three lineage III animal isolates. Hly alleles $1 \mathrm{c}, 4 \mathrm{a}$ and $4 \mathrm{~b}$ were unique to lineage III isolates and accounted for 5 of the 76 animal isolates $(6.6 \%)$ but only 1 of the 119 human isolates.

The actA PCR-RFLP assay used in this study differentiates two alleles, which are characterized by the presence of either three or four proline-rich repeats for act $A$ allele 3 or 4 , respectively. No significant differences in act $A$ allele distributions were found among the three categories of isolates (human epidemic, human sporadic and animal) (Table 3). However, the frequencies of the two act $A$ alleles differed significantly among the three lineages $\left(P<0 \cdot 0001 ; 3 \times 2 \chi^{2}\right.$ test $)$. act $A$ allele 4 was 
Table 4. SID for various typing methods

\begin{tabular}{|lcc|}
\hline \multirow{2}{*}{ Typing method } & \multicolumn{2}{c|}{ SID } \\
\cline { 2 - 3 } & Animal isolates & Human isolates \\
\hline Ribotyping only & 0.867 & 0.857 \\
Ribotyping and hly & 0.877 & 0.860 \\
Ribotyping and actA & 0.909 & 0.900 \\
Ribotyping, hly and & 0.916 & 0.904 \\
actA & & 0.703 \\
hly and actA & 0.737 & \\
\hline
\end{tabular}

found in $39 \cdot 6,92$ and $100 \%$ of the clinical isolates in lineages I, II and III, respectively.

\section{SID}

SID was calculated for each typing method, as well as for all possible combinations of different typing methods used (Table 4). The numerical value of this index $(D)$ indicates the suitability of a given method for differentiating strains by estimating the probability that two unrelated strains are differentiated by a given typing method (Hunter \& Gaston, 1988). As the numerical index approaches the maximum value of $D=1$ (representing $100 \%$ discriminatory ability of a method), the higher the probability that a given method will be able to discriminate between two unrelated strains. SID for automated ribotyping of either human or animal isolates was very similar $(0.867$ and 0.857 , respectively). When ribotyping was combined with allelic typing of both $\operatorname{act} A$ and $h l y$, the index of discrimination increased to 0.916 and 0.904 for animal or human isolates, respectively (Table 4).

\section{DISCUSSION}

We have defined specific subsets and clonal groups of $L$. monocytogenes that differ in their associations with animal and human listeriosis cases and outbreaks, and thus may show host specificities or unique pathogenic potentials. We have also demonstrated that ribotyping, particularly in conjunction with PCR-RFLP of hly and act $A$ virulence genes, provides an effective means for differentiating and characterizing L. monocytogenes isolates. The strains used in this study compose a collection of genetically well characterized human and animal L. monocytogenes isolates which should provide a valuable resource for further evaluation of typing and detection methods and for additional investigations of the role of strain diversity in L. monocytogenes pathogenesis and virulence.

\section{Clonal structure of $L$. monocytogenes}

The combination of PCR-RFLP and ribotyping defined 39 distinctive L. monocytogenes strains (Table 2). We differentiated 8 bly alleles, 2 act $A$ alleles, 25 ribotypes, 8 ribotype fragment subsets and 3 lineages, thus illustrating this organism's diversity among animal and human isolates. Separation of L. monocytogenes into three lineages representing genetically distinctive subsets of this species is consistent with a variety of previous studies. Phylogenetic classification methodologies have categorized L. monocytogenes strains initially into two, then more recently, three, distinct lineages (Brosch et al., 1994; Graves et al., 1994; Gutekunst et al., 1992; Piffaretti et al., 1989; Rasmussen et al., 1991; Vines et al., 1992). The different methodologies have resulted in notably similar strain groupings based on serotype distribution to distinct lineages, with $a$ and $c$ serotypes falling into one subgroup and $b$ serotypes into another (Gutekunst et al., 1992). Rasmussen et al. (1995) first suggested the existence of a third lineage based on sequence comparisons for $h l y A$, iap and the gene encoding flagellin $(f l a A)$. The strains in this group correspond with those classified into our lineage III by ribotyping and virulence gene alleles (Wiedmann et al., 1997). The clonal structure of the L. monocytogenes strains examined in this study is illustrated in Fig. 1. Each of the three lineages is divided into multiple ribotype fragment subsets, which represent groups sharing common ribotype fragments (Bruce et al., 1995). The number of ribotypes found in each subset and the positions of the three specific ribotypes with frequencies $>10 \%$ among our isolates are also indicated in Fig. 1. DUP-1042, the most common ribotype found among all isolate groups in this study, was responsible for four listeriosis epidemics (Table 1). DUP-1038, which was significantly more common in human epidemic cases than in animal or human sporadic cases, was responsible for five listeriosis epidemics (Table 1). While DUP-1030 was significantly more common among animal cases than among human cases, it was responsible for a human listeriosis outbreak in Carlisle in 1981.

\section{Indications for host specificities and differences in pathogenic potential}

The pathogenic potential of bacterial pathogens in various hosts has been demonstrated to be nonrandomly distributed along phylogenetic lines in many species (Musser et al., 1989; Selander \& Musser, 1990; Selander et al., 1987). For example, host specificities among clonal subsets have been described for humans and for different animal species in Staphylococcus aureus (Musser \& Selander, 1990), for different animal species in Bordetella spp. (Musser et al., 1987) and for cattle and sheep in P. haemolytica (Davies et al., 1997). Clonal groups with unique virulence potentials have also been reported for a variety of bacterial pathogens including Haemophilus influenzae (Quentin et al., 1990) and Streptococcus agalactiae (Helmig et al., 1993; Musser et al., 1989; Quentin et al., 1995).

We evaluated frequency distributions of five different genetic characteristics ( $h l y$ allele, act $A$ allele, ribotype, ribotype fragment subset and lineage) among three categories of L. monocytogenes isolates (human spor- 


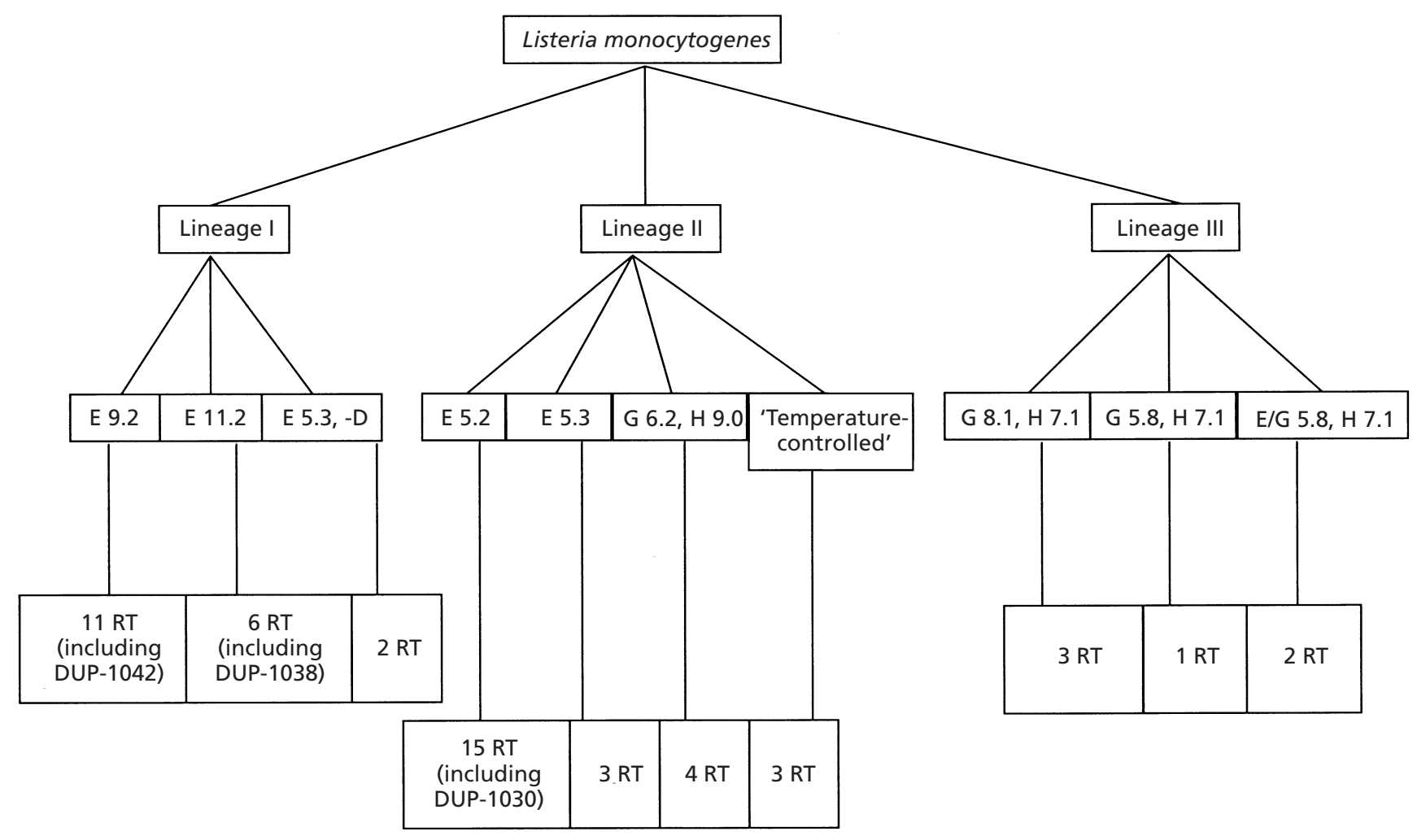

Fig. 1. Clonal structure of L. monocytogenes. L. monocytogenes consists of three lineages (Wiedmann et al., 1997), each of which is divided into multiple ribotype fragment subsets. Ribotype (RT) fragment subsets represent groups of ribotypes which share common ribotype fragments (Bruce et al., 1995). The number of ribotypes found in each subset is also indicated. The positions of the three specific ribotypes (DUP-1042, DUP-1038 and DUP-1030) with frequencies $>10 \%$ among our isolates are also indicated.

adic, human epidemic and animal sources) to assess the possibilities of host specificities or unique pathogenic potentials among specific subsets or clonal groups of this organism. The separation into human sporadic and epidemic isolates may not always be accurate since some apparent sporadic cases may actually represent unrecognized epidemics. We thus focused our initial analyses on comparing the frequency of animal isolates to all human isolates (sporadic and epidemic). Our results suggest that L. monocytogenes is a bacterial pathogen with non-random distributions of host specificities and pathogenic potentials among different clonal groups. Ribotypes or specific ribotype fragments that differentiate ribotype fragment subsets, appear to be good markers for L. monocytogenes clones that vary in virulence for particular host species.

Previous studies have shown that human epidemic $L$. monocytogenes isolates represent two unique clones of this species (Piffaretti et al., 1989), which are equivalent to ribotypes DUP-1038 and DUP-1042. In our study, human epidemic isolates showed a significant predominance of ribotype DUP-1038. The significant predominance of ribotype fragment subset E 11.2 among human epidemic isolates can be explained by the fact that DUP1038 classifies into this subset. Our work confirms the unique position of human epidemic isolates and expands upon the findings of previous studies by showing that ribotype DUP-1038 is indeed significantly more common among human epidemic isolates than among human sporadic isolates. DUP-1038 occurs less frequently, but with an approximately equal frequency, among human sporadic cases $(10.5 \%)$ and animal cases $(11.8 \%)$. With one exception, all DUP-1038 and DUP-1042 isolates were found to carry bly allele 1 , which is unique to lineage I strains and occurs in $98 \%$ of lineage I isolates. Ribotypes DUP-1038 and DUP-1042 include isolates from eight of the nine epidemic outbreaks listed in Table 1. These two epidemic clones also account for a large proportion $(41 \cdot 0 \%)$ of human sporadic listeriosis cases.

The human epidemic clone DUP-1042 is not significantly more common among human isolates as compared to animal isolates. Therefore, the occurrence of this clone in three human listeriosis outbreaks might not be indicative of a unique pathogenic potential, but might rather reflect the widespread distribution of this common L. monocytogenes ribotype. On the other hand, our data suggest that the predominance of DUP-1038 in human epidemics is not a consequence of the common occurrence of this L. monocytogenes strain. This strain is thus likely to be characterized by a unique potential to cause human food-borne listeriosis, possibly as a result of increased human virulence or an increased ability to 
survive and multiply in contaminated foods (Piffaretti et al., 1989). All epidemic DUP-1038 isolates have been characterized by the presence of actA allele 3, with the exception of one of two isolates from the 1985 Los Angeles outbreak. Representatives of this clone have been characterized by the presence of a unique form of act $A$ allele 3 which is not phosphorylated inside host cells (Wiedmann et al., 1997). Based on this observation and the fact that DUP-1038 shows only minimal allelic variation in act $A$ and $b l y$, we hypothesize that this ribotype represents a newly evolved clone with a unique ability to cause human listeriosis epidemics. This situation is reminiscent of Streptococcus agalactiae, where a single multilocus enzyme electrophoresis type (ET) is responsible for most of the serious human diseases caused by strains expressing the type III polysaccharide antigen. This ET is also characterized by the absence of allelic variations, thus suggesting that it represents a recently evolved clone (Musser et al., 1989).

Among the three major genetic lineages of L. monocytogenes, human isolates were significantly more frequently classified into lineage I than animal isolates. This lineage also contains the human epidemic clones. Thus, strains in this lineage appear more likely to cause human epidemic and sporadic listeriosis than strains classified into the other two lineages. Our results are consistent with previous studies, which indicate that certain L. monocytogenes subtypes are more commonly responsible for human listeriosis cases and outbreaks. For example, of 144 human isolates from sporadic cases serotyped by the CDC in $1986,30 \%$ were $1 / 2 \mathrm{a}, 32 \%$ were $1 / 2 \mathrm{~b}$ and $34 \%$ were $4 \mathrm{~b}$ (Schwartz et al., 1989). Of 1363 human isolates collected in the UK, $15 \%$ were $1 / 2 \mathrm{a}, 10 \%$ were $1 / 2 \mathrm{~b}$ and $64 \%$ were $4 \mathrm{~b}$ (McLauchlin, 1990). Serotypes $1 / 2 b$ and $4 b$, which are classified into lineage I, thus represent the majority of human isolates. Furthermore, Vines et al. (1992) reported that perinatal listeriosis is caused more frequently by strains equivalent to those in our lineage I than by strains in lineage II.

Classification into lineage III was significantly more common for animal isolates $(10.5 \%)$ than for human isolates $(0 \cdot 8 \%)$. While animal isolates were found in all three lineages, human isolates were confined almost entirely to lineages I and II. We interpret these data as an indication that lineage III strains represent a clonal group of L. monocytogenes with possible host specificity for non-primate mammals and with limited virulence in humans. Host factors play a key role in virulence of bacterial pathogens. The single human case caused by a lineage III strain may represent an infection of a highly susceptible individual and may not reflect the virulence characteristics of the bacterial isolate. Unfortunately, no detailed patient information was available for this isolate.

Lineage III strains were previously shown to be genetically distinct from lineage I and II strains as well as from the non-pathogenic species Listeria innocua (Bruce et al., 1995; Wiedmann et al., 1997) and thus this lineage may represent a distinct subset, and perhaps subspecies, of L. monocytogenes. Specifically, lineage III strains have unique $16 \mathrm{~S}$ rRNA sequences and have a distinctive ribotype fragment of $7 \cdot 1 \mathrm{~kb}$ (designated $\mathrm{H} 7.1$ ), which is not present in L. monocytogenes strains of lineages I and II, nor in L. innocua (Bruce et al., 1995; Wiedmann et al., 1997). Strains in two of the ribotype fragment subsets found in lineage III are rhamnose-negative, although L. monocytogenes is characteristically rhamnose-positive.

\section{Allelic variation in actA and $h l y$}

Acquisition or loss of genes encoding virulence determinants or mutations leading to new virulence gene alleles may alter host specificities and/or pathogenic potentials among clonal subsets of bacterial pathogens. For example, in Staphylococcus aureus, strains bearing distinct allelic variants of the gene encoding enterotoxin $\mathrm{C}$ differ in their ability to stimulate ovine, bovine and human T-cells (Marr et al., 1993) and allelic variants of the toxic shock syndrome toxin also differ in their virulence properties (Lee et al., 1992).

In a previous study (Wiedmann et al., 1997), we hypothesized that allelic variation in virulence genes, and specifically in act A, may play a role in determining the unique pathogenic potential of the epidemic $L$. monocytogenes clone DUP-1038. It is also possible that these unique alleles are not solely responsible for virulence differences among strains and that they may instead be markers for the presence or absence of yet uncharacterized virulence genes responsible for host specificity or unique pathogenic potential among $L$. monocytogenes strains. In this study, we probed correlations between bly and actA allele frequencies and clinical histories (i.e. human or animal source) of $L$. monocytogenes isolates.

We did not find any significant differences in the distribution of act A alleles 3 and 4 among human epidemic, human sporadic and animal isolates. Significant differences existed for the frequencies of act $A$ alleles 3 and 4 among the three lineages, however (data not shown; $P<0 \cdot 001)$. All $(100 \%)$ lineage III clinical isolates and $92 \%$ of lineage II isolates carried act A allele 4. Interestingly, of 12 non-clinical lineage III isolates, 11 carried act $A$ allele 3 (data not shown). We therefore hypothesize that lineage III strains carrying act $A$ allele 3 are virulence-attenuated for both humans and animals. Indeed, previous work has shown that at least two lineage III isolates with actA allele 3 are virulenceattenuated (Chakraborty et al., 1994; Wiedmann et al., 1997). DNA sequencing further revealed that the actA alleles from these two isolates and from an attenuated lineage II isolate share an identical signature sequence with glutamic acid at position 375 and three proline-rich repeats (i.e. act $A$ allele 3 ). This combination was unique in comparison with 11 other act A allele 3 genes sequenced (Wiedmann et al., 1997). Since previous DNA sequencing studies have indicated significant heterogeneity within each of the actA alleles (Wiedmann et al., 1997), it is important to note that the PCR-RFLP method 
used in this study only differentiates two actA alleles, indicating the presence of either three or four prolinerich repeats. Since the presence of the unphosphorylated ActA protein unique to the epidemic clone DUP-1038 can only be detected by actA DNA sequencing or allelespecific PCR (Wiedmann et al., 1997), this study did not investigate the occurrence of this unique allele among clinical isolates. However, our data provide indirect evidence suggesting that unphosphorylated ActA, which appears to be unique to all DUP-1038 isolates (previously designated dd0647), might play an important role in human virulence since DUP-1038 shows significant predominance in human epidemic isolates (Table 3).

\section{Discriminatory ability of automated ribotyping and PCR-RFLP of actA and hly}

Reliable and discriminatory typing methods are crucial for studies of population genetics as well as for epidemiological investigations. We therefore evaluated the ability of the different typing methods used in this study to permit sensitive discrimination of clinical $L$. monocytogenes isolates. SID (Hunter \& Gaston, 1988), a quantitative measure of the discriminatory ability of a given typing method, showed a high discriminatory power for the combination of ribotyping and PCRRFLP of actA and hly (SID of 0.92 and 0.90 for animal and human isolates, respectively). As a single typing method from among these three approaches, ribotyping showed the highest discrimination (SID of 0.87 and 0.86 for animal and human isolates, respectively). In comparison, in the WHO International Multicentre $L$. monocytogenes subtyping study, the SID values achieved by various methods were as follows: ribotyping, $0 \cdot 83-0 \cdot 88 ; \lambda$ RFLP, $0 \cdot 81-0 \cdot 91$ (Swaminathan et al., 1996); serotyping, $0 \cdot 70$; multilocus enzyme electrophoresis, 0.83-0.93 (Caugant et al., 1996). We conclude that automated ribotyping provides a high level of discrimination for both human and animal L. monocytogenes isolates. Strain discrimination by ribotyping is further enhanced by the addition of PCR-RFLP analyses of act $A$ and hly.

\section{Conclusions}

Our data indicate the existence of distinct L. monocytogenes clonal groups, which appear to differ in their pathogenic potential and their host specificity. Furthermore, these different clonal groups may also differ in their adaptation to different ecological niches (Boerlin \& Piffaretti, 1991). These findings may have major implications for the development of rational regulations and effective control measures for food-borne listeriosis, which should be specifically directed against pathogenic bacterial clones responsible for human infections. Our database of genetically well characterized clinical $L$. monocytogenes isolates also provides a basis for future surveillance programmes for monitoring the occurrence of listerial clones and their frequencies in human and animal listeriosis. Such database development can contribute to comprehensive public health programmes since the information may be used to help predict and detect new clones with unique host ranges and pathogenic potentials.

\section{ACKNOWLEDGEMENTS}

Some of the material described in this manuscript is based upon work supported by the USDA National Research Initiative under Award No.99-35201-8074. The authors would like to thank M. Louie (Sunnybrook Health Science Centre, University of Toronto, Ontario, Canada), B. Swaminathan and L. Graves (Centers for Disease Control, Atlanta, Georgia), Dr J. Harvey (Department of Health Services, Los Angeles, California) and Qualicon (Wilmington, Delaware) for providing human $L$. monocytogenes isolates. The authors also thank Dr Ynte Schukken for help with statistical analyses.

\section{REFERENCES}

Bille, J. (1990). Epidemiology of human listeriosis in Europe, with special reference to Swiss outbreak. In Foodborne Listeriosis, pp. 71-74. Amsterdam : Elsevier.

Bille, J. \& Rocourt, J. (1996). WHO International Multicentre Listeria monocytogenes subtyping study: rationale and set-up of the study. Int J Food Microbiol 32, 251-262.

Boerlin, P. \& Piffaretti, J.-C. (1991). Typing of human, food and environmental isolates of Listeria monocytogenes by multilocus enzyme electrophoresis. Appl Environ Microbiol 57, 1624-1629.

Brosch, R., Chen, J. \& Luchansky, J. B. (1994). Pulsed-field fingerprinting of listeriae: identification of genomic divisions for Listeria monocytogenes and their correlation with serovar. Appl Environ Microbiol 60, 2584-2592.

Bruce, J. (1996). Automated system rapidly identifies and characterizes micro-organisms in food. Food Technol 50, 77-81.

Bruce, J. L., Hubner, R. J., Cole, E. M., McDowell, C. I. \& Webster, J. A. (1995). Sets of EcoRI fragments containing rRNA sequences are conserved among different strains of Listeria monocytogenes. Proc Natl Acad Sci US A 92, 5229-5233.

Carbonelle, B., Cottin, J., Parvery, F., Chambreuil, G., Kouyoumdjian, S., Le Lirzin, M., Cordier, G. \& Vincent, F. (1978). Epidemie de listeriose dans l'ouest de la France (1975-1976). Rev Epidemiol Sante Publique 26, 451-467.

Caugant, D. A., Ashton, F. E., Bibb, W. F., Boerlin, P., Donachie, W., Low, C., Gilmour, A., Harvey, J. \& Norrung, B. (1996). Multilocus enzyme electrophoresis for characterization of Listeria monocytogenes isolates: results of an international comparative study. Int J Food Microbiol 32, 301-310.

Chakraborty, T., Ebel, F., Wehland, J., Dufrenne, J. \& Notermans, S. (1994). Naturally occurring virulence-attenuated isolates of Listeria monocytogenes capable of inducing long term protection against infection by virulent strains of homologous and heterologous serotypes. FEMS Immunol Med Microbiol 10, 1-10.

Davies, R. L., Arkinsaw, S. \& Selander, R. K. (1997). Evolutionary genetics of Pasteurella haemolytica isolates recovered from cattle and sheep. Infect Immun 65, 3585-3593.

Farber, J. M. \& Peterkin, P. I. (1991). Listeria monocytogenes, a food-borne pathogen. Microbiol Rev 55, 476-511.

Fleming, D. W., Cochi, S. L., MacDonald, K. L. \& 7 other authors (1985). Pasteurized milk as a vehicle of infection in an outbreak of listeriosis. N Engl J Med 312, 404-407.

Graves, L. M., Swaminathan, B., Reeves, M. W., Hunter, S. B., Weaver, R. E., Plikaytis, B. D. \& Schuchat, A. (1994). Comparison of ribotyping and multilocus enzyme electrophoresis for sub- 
typing Listeria monocytogenes isolates. J Clin Microbiol 32, 2936-2943.

Gutekunst, K. A., Holloway, B. P. \& Carlone, G. M. (1992). DNA sequence heterogeneity in the gene encoding a 60-kilodalton extracellular protein of Listeria monocytogenes and other Listeria species. Can J Microbiol 38, 865-870.

Helmig, R., Uldbjerg, N., Boris, J. \& Kilian, M. (1993). Clonal analysis of Streptococcus agalactiae isolated from infants with neonatal sepsis or meningitis and their mothers and from healthy pregnant women. J Infect Dis 168, 904-909.

Ho, J. L., Shands, K. N., Friedland, G., Eckind, P. \& Fraser, D. W. (1986). An outbreak of type $4 \mathrm{~b}$ Listeria monocytogenes infection involving patients from eight Boston hospitals. Arch Intern Med 146, 520-524.

Hof, H. \& Rocourt, J. (1992). Is any strain of Listeria monocytogenes detected in food a health risk? Int J Food Microbiol 16, 173-182.

Hunter, P. \& Gaston, M. A. (1988). Numerical index of the discriminatory ability of typing systems: an application of Simpson's index of diversity. J Clin Microbiol 26, 2465-2466.

Kapur, V., Sischo, W. M., Greer, R. S., Whittam, T. \& Musser, J. M. (1995). Molecular population genetic analysis of Staphylococcus aureus recovered from cows. J Clin Microbiol 33, 376-380.

Lee, P. K., Kreiswirth, B. N., Deringer, J. R., Projan, S. J., Eisner, W., Smith, B. L., Carlson, E., Novick, R. P. \& Schlievert, P. M. (1992). Nucleotide sequence and biological properties of toxic shock syndrome toxin 1 from ovine- and bovine-associated Staphylococcus aureus. J Infect Dis 165, 1056-1063.

Linnan, M. J., Mascola, L., Lou, X. D. \& 12 other authors (1988). Epidemic listeriosis associated with Mexican-style cheese. N Engl J Med 319, 823-828.

Louie, M., Jayaratne, P., Luchsinger, I., Devenish, J., Yao, J., Schlech, W. \& Simor, I. (1996). Comparison of ribotyping, arbitrarily primed PCR and pulsed-field gel electrophoresis for molecular typing of Listeria monocytogenes. J Clin Micro 34, $15-19$.

Marr, J. C., Lyon, J. D., Roberson, J. R., Lupher, M., Davies, W. C. \& Bohach, G. A. (1993). Characterization of novel type C staphylococcal enterotoxins: biological and evolutionary implications. Infect Immun 61, 4254-4262.

McLauchlin, J. (1990). Distribution of serovars of Listeria monocytogenes isolated from different categories of patients with listeriosis. Eur J Clin Microbiol Infect Dis 9, 210-213.

McLauchlin, J., Audurier, A. \& Taylor, A. G. (1986). Aspects of the epidemiology of human Listeria monocytogenes infections in Britain 1967-1984; the use of serotyping and phage typing. J Med Microbiol 22, 367-377.

McLauchlin, J., Hall, S. M., Velani, S. K. \& Gilbert, R. J. (1991). Human listeriosis and paté: a possible association. Br Med J 303, 773-775.

Mead, P., Slutsker, L., Dietz, V., McCaig, L. F., Bresee, J. S., Shapiro, C., Griffin, P. M. \& Tauxe, R. V. (1999). Food-related illness and death in the United States. Emerg Infect Dis 5, 607-625.

Musser, J. M. \& Selander, R. K. (1990). Genetic analysis of natural populations of Staphylococcus aureus. In Molecular Biology of the Staphylococci, pp. 59-67. Edited by R. Novick \& R. A. Skurray. New York: VCH.

Musser, J. M., Bemis, D. A., Ishikawa, H. \& Selander, R. K. (1987). Clonal diversity and host distribution in Bordetella bronchiseptica. J Bacteriol 169, 2793-2803.
Musser, J. M., Mattingly, S. J., Quentin, R., Goudeau, A. \& Selander, R. K. (1989). Identification of a high-virulence clone of type III Streptococcus agalactiae (group B streptococcus) causing invasive neonatal disease. Proc Natl Acad Sci U S A 86, 4731-4735.

Piffaretti, J.-C., Kressebuch, H., Aeschenbacher, M., Bille, J., Bannerman, E., Musser, J. M., Selander, R. K. \& Rocourt, J. (1989). Genetic characterization of clones of the bacterium Listeria monocytogenes causing epidemic disease. Proc Natl Acad Sci U S A 86, 3818-3822.

Quentin, R., Goudeau, A., Wallace, R. J., Smith, A. L., Selander, R. K. \& Musser, J. M. (1990). Urogenital, maternal and neonatal isolates of Haemophilus influenzae: identification of unusually virulent serologically non-typable clone families and evidence for a new Haemophilus species. J Gen Microbiol 136, 1203-1209.

Quentin, R., Huet, H., Wang, F., Geslin, P., Goudeau, A. \& Selander, R. (1995). Characterization of Streptococcus agalactiae strains by multilocus enzyme genotype and serotype: identification of multiple virulent clone families that cause invasive neonatal disease. J Clin Microbiol 33, 2576-2581.

Rasmussen, O. F., Beck, T., Olsen, J. E., Dons, L. \& Rossen, L. (1991). Listeria monocytogenes isolates can be classified into two major types according to the sequence of the listeriolysin gene. Infect Immun 59, 3945-3951.

Rasmussen, O. F., Skouboe, P., Dons, L., Rosen, L. \& Olsen, J. E. (1995). Listeria monocytogenes exists in at least three evolutionary lines: evidence from flagellin, invasive associated protein and listeriolysin O genes. Microbiology 141, 2053-2061.

Schlech, W. F., Lavigne, P. M., Bortolussi, R. A. \& 8 other authors (1983). Epidemic listeriosis: evidence for transmission by food. $N$ Engl J Med 308, 203-206.

Schwartz, B., Hexter, D., Broome, C. V. \& 7 other authors (1989). Investigation of an outbreak of listeriosis: new hypotheses for the aetiology of epidemic Listeria monocytogenes infections. J Infect Dis 159, 680-685.

Selander, R. K. \& Musser, J. M. (1990). Population genetics of bacterial pathogenesis. In Molecular Basis of Bacterial Pathogenesis, pp. 11-36. Edited by B. H. Iglewski \& V. L. Clark. San Diego, CA: Academic Press.

Selander, R. K., Musser, J. M., Caugant, D. A., Gilmour, M. N. \& Whittam, T. S. (1987). Population genetics of pathogenic bacteria. Microb Pathog 3, 1-7.

Swaminathan, B., Hunter, S. B., Desmarchelier, P. M. \& 10 other authors (1996). WHO-sponsored international collaborative study to evaluate methods for subtyping Listeria monocytogenes: restriction fragment length polymorphism (RFLP) analysis using ribotyping and Southern blotting with two probes derived from L. monocytogenes chromosome. Int J Food Microbiol 32, 263-278.

Vines, A., Reeves, M. W., Hunter, S. \& Swaminathan, B. (1992). Restriction fragment length polymorphism in four virulenceassociated genes of Listeria monocytogenes. Res Microbiol 143, 281-294.

Wiedmann, M., Bruce, J. L., Keating, C., Johnson, A., McDonough, P. L. \& Batt, C. A. (1997). Ribotypes and virulence gene polymorphisms suggest three distinct Listeria monocytogenes lineages with differences in pathogenic potential. Infect Immun $\mathbf{6 5}$, 2707-2716.

Received 13 October 2000; revised 2 January 2001; accepted 16 January 2001. 